\title{
Effect of a structured, active, home-based cancer-treatment program for the management of patients on oral chemotherapy
}

This article was published in the following Dove Press journal:

Patient Preference and Adherence

25 June 2014

Number of times this article has been viewed

\section{S Bordonaro' \\ F Romano' \\ E Lanteri' \\ F Cappuccio' \\ $R$ Indorato ${ }^{2}$ \\ A Butera ${ }^{2}$ \\ A D'Angelo 3 \\ F Ferraù ${ }^{3}$ \\ P Tralongo'}

'Medical Oncology Unit, Umberto I Hospital, Rete Assistenza Oncologica, Siracusa, ${ }^{2}$ Medical Oncology Unit, S Giovanni di Dio Hospital, Agrigento, ${ }^{3}$ Medical Oncology Unit,

S Vincenzo Hospital, Taormina, Italy
Correspondence: Paolo Tralongo Medical Oncology Unit, Umberto I Hospital, RAO, Siracusa, Italy

Tel +3993172 4542

Email tralongo@raosr.it
Introduction: The advent of oral chemotherapy agents has had a strong impact on several aspects of the management of cancer patients, including survival rates, health-care expenditure, and health-related quality of life. However, access to care and adherence to oral chemotherapy are central to optimal outcomes.

Patients and methods: In this multicenter observational study, we assessed the effect of the "Active Home Care" initiative - a structured, active, home-based cancer-treatment program on quality of life, health-care utilization, and patient adherence and satisfaction using selfadministered questionnaires. Sixty-two patients treated with oral chemotherapy (capecitabine, vinorelbine, imatinib, sunitinib, sorafenib, temozolomide, ibandronate) were enrolled in the program. Weekly home visits were scheduled, each one with a trained nurse who delivered the home-based chemotherapy and reviewed patients' compliance and treatment toxicity. An oncologist evaluated patients and modified the dosage of oral chemotherapy based on toxicity reported during the previous cycle at bi-weekly visits.

Results: A total of 460 home visits were performed between April 2012 and February 2013. The Active Home Care initiative was associated with significant improvements in physical functioning and symptoms, and reductions in the access to cancer facilities. Satisfaction with oral chemotherapy and care received was high. All patients reported having taken their medications according to their prescription, and no patient reported difficulties in managing the oral chemotherapy regimen.

Conclusion: The Active Home Care program was associated with improvements in the quality of life of patients and caregivers, better adherence to treatment, and the effective management of therapy and cancer-related symptoms. Home-based cancer treatment may also optimize the utilization of health-care resources.

Keywords: advanced cancer, home-based care, quality of life, adherence, patient satisfaction, effective therapy management

\section{Introduction}

Over the past decades, the availability of new treatment options and advances in the prevention, earlier detection, and treatment of cancer have led to a substantial decrease in mortality rates from the disease. ${ }^{1}$ As such, most cancers are now regarded as chronic conditions that require long-term treatment and follow-up. At the same time, the decrease in cancer death rates, along with the aging of the population and the spread of cancer-causing behaviors such as smoking, has translated into an increased prevalence of cancer worldwide. ${ }^{2}$ Overall, increases in survival and cancer prevalence have resulted in a greater number of cancer patients needing long-term care, which poses significant challenges to clinical oncologists and oncology services. It should 
also be highlighted that quality of life is a primary goal in the management of patients with cancer, especially for those with advanced disease, since cancer therapies strongly affect both patients' and caregivers' quality of life. ${ }^{3-5}$ Therefore, the need for management strategies aimed at improving quality of life, clinical outcomes, and patient acceptability, as well as optimizing the utilization of health-care resources, is compelling. In this light, the use of oral cancer therapies, which is becoming increasingly common in clinical oncology, ${ }^{6,7}$ represents a promising therapeutic strategy. The advent of oral chemotherapy has had a strong impact on several aspects of the management of cancer patients, including survival rates, health-care expenditure, and health-related quality of life. ${ }^{8-10}$ As an example, the selective tyrosine kinase inhibitor imatinib mesylate has been shown to be highly effective in patients with unresectable or metastatic gastrointestinal stromal tumor - a cancer associated with unfavorable outcomes and resistance to conventional chemoor radiotherapy. ${ }^{11,12}$ Imatinib is also considered life saving for patients with chronic myeloid leukemia. Similarly, capecitabine and vinorelbine have made an important contribution to the treatment of advanced and/or metastatic breast, colon, gastric, and lung cancers. ${ }^{14-19}$

Although oral regimens offer the great convenience of treatment at home and avoidance of infusions that often require the placement of long-term venous access devices potentially associated with increased risk of infections and thrombotic complications, ${ }^{20}$ the efficacy of oral chemotherapy is largely dependent on patients' adherence to the prescribed protocol. ${ }^{21,22}$ In fact, self-administration may increase the likelihood of errors, including missing a dose, overdosing, or taking a dose at the wrong time, which in turn may lead to reduced efficacy, increased toxicity, and potential drugdrug interactions in patients taking other medications. ${ }^{21,23}$ Adherence rates for oral cancer therapy vary widely, ranging from less than $20 \%$ to $100 \%,{ }^{21}$ and nonadherence is known to be associated with greater use of health-care resources and costs due to more frequent physician visits, higher hospitalization rates, and longer hospital stays. ${ }^{24,25}$ Several factors may contribute to poor adherence, eg, individual patient characteristics, features of the disease and the treatment regimen, and aspects of the medical care system. ${ }^{22}$ However, patient education by physicians, nurses, and other health-care providers, as well as ease of access to health care, may substantially improve adherence. ${ }^{26}$ As a consequence of the increased availability and use of oral chemotherapy, in recent years, the need for a shift from inpatient to home-based care strategies has become apparent.
Home-based health-promotion programs have been shown to offer clinical benefits in a range of clinical settings and to be cost-effective. ${ }^{27,28}$ In the present study, we sought to assess the effect of a structured, active, home-based cancer treatment program - on quality of life, health-care utilization, and patient adherence and satisfaction in a cohort of patients treated with oral chemotherapy.

\section{Patients and methods}

This was an observational, longitudinal, multicenter study. Participants were selected among adult ( $\geq 18$ years old) cancer patients being seen at three oncology referral centers in Sicily, Italy (Rete Assistenza Oncologica, Siracusa; Ospedale S Giovanni di Dio, Agrigento; and Ospedale S Vincenzo, Taormina) between April 2012 and February 2013. All patients on an oral chemotherapy regimen were asked to take part in the study, and those willing to participate were enrolled after providing informed consent. In the participating centers, a home-based cancer treatment program (the Active Home Care project) has been implemented for patients treated with oral chemotherapy, and these patients are routinely followed-up in their homes. The study was approved by the local ethics committee. Participation in the study did not affect the management of patients.

\section{Home-based cancer-treatment program}

In the home-based cancer program offered by the participating centers, weekly home visits are scheduled with a trained nurse who delivers the home-based chemotherapy and reviews patients' compliance and treatment toxicity. An oncologist evaluates patients and modifies the dosage of oral chemotherapy based on toxicity during the previous cycle at bi-weekly patient home visits. A telephone number is available 24 hours a day, 7 days a week for patients' emergency calls.

\section{Assessments}

For the purpose of this study, patients were asked to report any unplanned use of primary care, admissions to the emergency department, or hospitalizations. Patient quality of life was measured at baseline and after 3 months or two chemotherapy cycles (whichever occurred first) using the 30-item European Organisation for Research and Treatment of Cancer (EORTC) QLQ-C30 questionnaire, version 3.0, ${ }^{29}$ which includes several functional scales (physical, emotional, cognitive, social), a global-health-status quality-of-life scale, and single measures of symptom severity (fatigue, nausea, vomiting, pain, dyspnea, sleep disturbances, appetite loss, constipation, 
diarrhea, financial difficulties). High scores indicate better health-related quality of life for the global health status and functioning scales, and worse health-related quality of life for the symptom scales. According to the questionnaire scoring manual, a change of ten points or more is considered a moderate-to-large clinically significant change. ${ }^{29}$

Acceptability of oral chemotherapy was evaluated by a self-administered questionnaire specifically designed to identify the adherence to therapy and degree of satisfaction with oral chemotherapy compared with intravenous therapies. The questionnaire included several items to evaluate patients' general satisfaction with the health care and information received, adherence to therapy, opinion on oral chemotherapy (convenience, efficacy, and advantages associated with the oral regimen, such as less time spent in hospital and having more time to spend with family and friends), and difficulties in managing the drug regimen.

Another questionnaire was developed to provide an estimate of the clinical, social, and economic impact of the home-based care. The questionnaire assessed patients' and caregivers' overall satisfaction with the program (on a five-point scale from 1 [least satisfied] to 5 [most satisfied]), presence and type of cancer symptoms and/or treatmentrelated adverse events, psychological stress associated with the underlying disease, use of health-care resources (primary care or emergency department visits), and the need for assistance from a family member/caregiver, at baseline and after 6 months.

Data were assessed for normality with the Shapiro-Wilk test. The Wilcoxon signed-rank test was used to compare changes in quality of life. Descriptive statistics were used to describe the results of the questionnaire on the acceptability of other variables. A $P$-value of $<0.05$ was considered statistically significant. All statistical analyses were performed using SPSS (v 18.0, IBM Corp, Armonk, NY, USA).

\section{Results}

Sixty-two patients (26 males) with a mean age of 67.8 years (range 33-83 years) on oral chemotherapy were enrolled in the Active Home Care project in the study. More than half of the patients $(56 \%)$ were 70 years of age or older. Of the participating patients, $27.4 \%$ were affected by breast cancer, $24.1 \%$ by colon cancer, $19.3 \%$ by lung cancer, $6.4 \%$ by renal cancer, $6.4 \%$ by astrocytoma, $3.2 \%$ by hepatocellular carcinoma, $3.2 \%$ by gastrointestinal stromal tumor, $1.6 \%$ by pancreas cancer, $1.6 \%$ by uterine metastatic cancer, $1.6 \%$ by ovarian metastatic cancer, $1.6 \%$ by Kaposi's sarcoma, $1.6 \%$ by multiple myeloma, $1.6 \%$ by bladder cancer, and $1.6 \%$ by occult primary cancer. One patient had breast and colon cancer, while another patient had breast and uterine cancer. Therapies consisted of the anticancer drugs most often used in oral formulations (capecitabine, vinorelbine, imatinib, sunitinib, sorafenib, temozolomide, and ibandronate).

A total of 460 home visits were performed during the period considered. Only nine hospital admissions were recorded. Of these, five were for emergency care (emergency department) due to reasons not directly related to the primary condition and/or cancer treatment, and the remaining four were day-hospital admissions, none of which required subsequent hospitalization.

\section{Quality of life questionnaire}

Fifty-six patients completed the EORTC quality of life questionnaire (QLQ-C30) at baseline and 3 months (or two chemotherapy cycles) after enrollment in the program. Changes in EORTC QLQ-C30 scores are shown in Table 1. Significant improvements $(P<0.05)$ were seen in the symptom (fatigue, pain, nausea, and vomiting) and physical-functioning scales and in several individual items (dyspnea, sleep disturbances, appetite loss, and constipation) (Table 1). Of note, moderate-to-large clinically significant changes ( $\geq 10$ points) were observed in physical functioning, fatigue, nausea and vomiting, appetite loss, and constipation (Figure 1). There was also a trend toward an improvement in global quality of life and in most of the other scales (Table 1).

\section{Acceptability of oral chemotherapy}

Acceptability of oral chemotherapy was high. All patients considered the information received on the oral regimen to be complete and exhaustive, and thought that being on oral therapy was more advantageous than being on intravenous therapies, both in terms of time saved (less time spent in hospital) and of having more time to spend with family and friends. Also, all patients thought that an oral regimen was more convenient, since no bulky infusion devices are involved. Fifty-five patients $(88.7 \%$ ) considered oral chemotherapy to be as effective as chemotherapy infusion regimens, and $80.6 \%$ thought that an oral regimen could have fewer side effects than other therapies. Sixty patients $(96.7 \%)$ considered the home monitoring of compliance to treatment, patient health status, and possible adverse events by a nurse and/or an oncologist to be useful. No patient reported difficulties in managing the oral regimen, and all of them reported having taken their medications according to the indications provided by the physician. Forty-two patients (67\%) needed help from a caregiver to take their medications. 
Table I Differences between European Organisation for Research and Treatment of Cancer QLQ-C30 scores at baseline and at the 3-month assessment

\begin{tabular}{|c|c|c|c|c|c|}
\hline \multirow[t]{2}{*}{ Scale } & \multicolumn{2}{|c|}{ Baseline } & \multicolumn{2}{|c|}{ After 3 months } & \multirow[t]{2}{*}{$P$-value } \\
\hline & Mean & Interquartile range & Mean & Interquartile range & \\
\hline Global health status/QoL & 48.5 & $41.6-66.6$ & 64.2 & $41.6-66.6$ & 0.307 \\
\hline Physical functioning & 50.3 & $40.0-60.0$ & 61.4 & $40.0-80.0$ & 0.002 \\
\hline Role functioning & 76.9 & $66.6-83.3$ & 74.0 & $66.6-74.0$ & 0.599 \\
\hline Emotional functioning & 66.6 & $50.0-76.6$ & 66.6 & $50.0-91.6$ & 0.417 \\
\hline Cognitive functioning & 66.6 & $50.0-79.9$ & 66.6 & $50.0-83.3$ & 0.148 \\
\hline Social functioning & 66.6 & $51.9-95.3$ & 66.6 & $57.5-95.3$ & 0.349 \\
\hline Fatigue & 30.1 & $6.9-55.3$ & 8.3 & $0.0-30.1$ & 0.002 \\
\hline Nausea and vomiting & 25.0 & $14.4-50.0$ & 0.0 & $0.0-25.0$ & 0.001 \\
\hline Pain & 23.4 & $12.4-33.3$ & 16.6 & $0.0-32.2$ & 0.011 \\
\hline Dyspnea & 33.3 & $28.2-38.3$ & 28.2 & $16.7-33.3$ & 0.018 \\
\hline Insomnia & 33.3 & $16.6-33.3$ & 16.7 & $0.0-33.3$ & 0.004 \\
\hline Appetite loss & 44.8 & $34.9-66.6$ & 32.1 & $0.0-38.2$ & $<0.00 \mathrm{I}$ \\
\hline Constipation & 33.3 & $30.6-44.6$ & 30.6 & $0.0-33.3$ & $<0.00$ I \\
\hline Diarrhea & 0.0 & $0.0-0.0$ & 0.0 & $0.0-0.0$ & 0.366 \\
\hline Financial difficulties & 8.4 & $0.0-16.9$ & 8.4 & $0.0-16.9$ & 0.948 \\
\hline
\end{tabular}

Abbreviations: QLQ-C30, quality of life questionnaire; QoL, quality of life.

\section{Clinical, social, and economic impact of the home-based care}

Twenty-nine patients (46.8\%) completed the self-administered questionnaire for the assessment of the clinical, social, and economic impact of the home-based care, at baseline and after 6 months. After 6 months, the mean ratings of patients' and caregivers' overall satisfaction with the program on a fivepoint scale were 4.0 and 4.4 , respectively. The percentage of patients reporting cancer- and/or treatment-related symptoms decreased from $68.9 \%$ at baseline to $62.1 \%$ after 6 months. Fatigue and sleep disturbances were the most common symptoms, followed by pain, diarrhea and nausea, fever, vomiting, and dysphagia. The evaluation of symptoms related to the

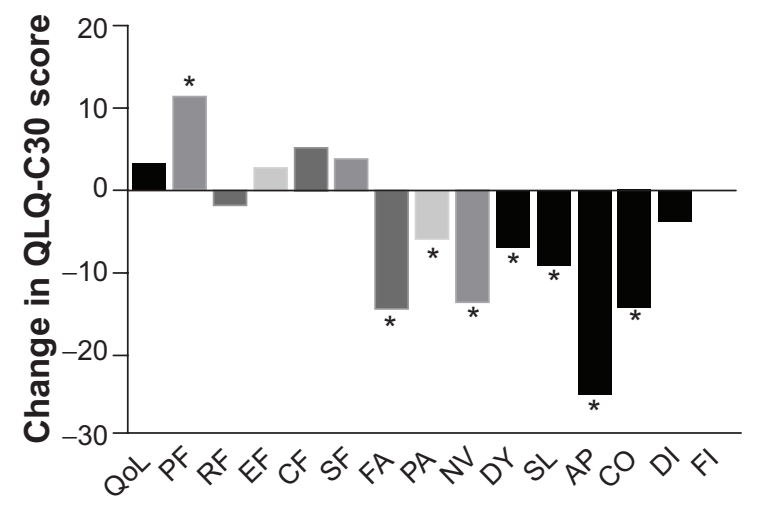

Figure I Mean change from baseline in the European Organisation for Research and Treatment of Cancer QLQ-C30 questionnaire after 3 months.

Note: *Significant difference from baseline $(P<0.05)$.

Abbreviations: AP, appetite loss; CF, cognitive functioning; $C O$, constipation; DI, diarrhea; DY, dyspnea; EF, emotional functioning; FA, fatigue; Fl, financial difficulties; $\mathrm{NV}$, nausea and vomiting; PA, pain; PF, physical functioning; QLQ-C30, quality of life questionnaire; QoL, global quality of life; RF, role functioning; SF, social functioning; $\mathrm{SL}$, insomnia. disease or the treatment is reported in Figure 2. All patients reported having been informed on treatment-related side effects potentially associated with the medication they were taking. After 6 months, the percentage of patients who saw their primary-care physician at least once during the month prior to completion of the questionnaire was numerically lower than at baseline ( $65.5 \%$ vs $86.2 \%$, respectively). In most cases, visits to the primary-care physician were arranged to ask for a generic drug prescription or to inform the physician on the patient's health conditions. Only three patients reported having seen their primary-care physician to ask for a prescription related to the chemotherapy protocol. Of note, the majority of patients $(82.7 \%$ at baseline and $86.2 \%$ at 6 months) would have needed to be accompanied to hospital visits by a family member or friend who, in most cases, had to take time off from work to do so. With the active homebased cancer treatment, patients with a poor performance status have been able to continue the oral treatment.

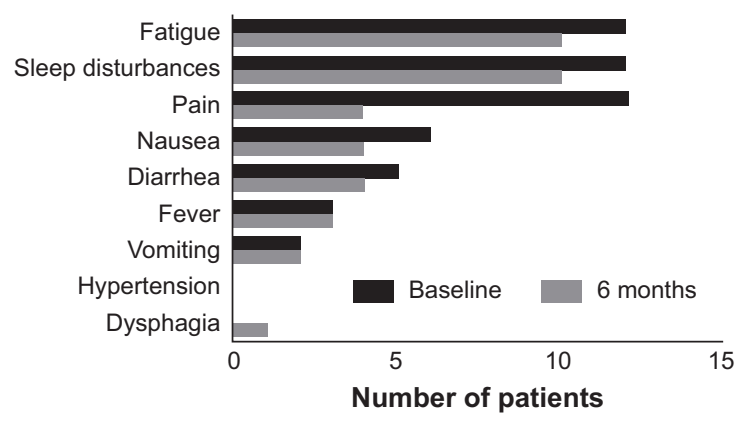

Figure 2 Cancer- and/or treatment-related symptoms reported by patients at baseline and after 6 months. 
With regard to psychological stress associated with the underlying disease, the number of patients who reported being scared slightly increased, from 17 at baseline to 19 at 6 months. Specifically, patients were afraid that cancerand/or treatment-related symptoms such as pain could become impossible to control; expressed concerns about losing their self-sufficiency or becoming a burden for their families; and were scared of dying, of losing their mental self-control or social role, or of being rejected by their families.

\section{Discussion}

Over the past decades, the availability of oral chemotherapy agents and the increase in the number of cancer patients requiring long-term care have led to a shift in the focus of care for patients with advanced cancer from institution-based to home-based models. We undertook the study reported here to evaluate the impact of an active, home-based, cancer-treatment program on quality of life, health-care utilization, and patient adherence and satisfaction in a cohort of patients treated with oral chemotherapy.

Participants reported significant improvements in physical functioning and cancer- and/or treatment-related symptoms. Moderate-to-large clinically significant changes were observed in physical functioning, fatigue, nausea and vomiting, appetite loss, and constipation (Figure 1). The Active Home Care project appeared to have a particularly positive effect on the appetite-loss score, which improved by about 25 points. The effective management of symptoms is a major priority in the care of cancer patients. Fatigue is highly prevalent among cancer patients, being present in $70 \%-100 \%$ of patients. ${ }^{30,31}$ Similarly, up to $64 \%$ of cancer patients experience pain. ${ }^{32}$ Cancer anorexia is a detrimental and highly prevalent symptom that, especially if associated with cancer cachexia, may lead to further worsening of patients' health status. ${ }^{33}$ It has been suggested that the best approach to the so-called cancer anorexia-cachexia syndrome should include optimal symptom management and careful psychosocial counselling. ${ }^{34}$ Overall, the observed improvement in physical functioning and symptoms such as pain, fatigue, and appetite loss may be related to easier access to care and better communication between patients and health-care providers, which facilitated earlier detection and management of symptoms.

Further, it has been shown that cancer patients receiving home-based care are more confident in dealing with the effects of their disease. ${ }^{35}$ Accordingly, a trend toward an improvement in global quality of life and emotional, cognitive, and social functioning was also observed. Preference for oral chemotherapy over parenteral treatments was high among patients. These results are in accordance with previous reports in the literature indicating that a large proportion of cancer patients prefer oral chemotherapy over intravenous regimens. ${ }^{36,37}$ Further, most patients considered oral chemotherapy to be as effective as other treatments and associated with fewer side effects. It should be acknowledged, however, that oral equivalents have side effects that are similar to their parenteral counterparts. ${ }^{13}$ In this light, patient education is essential to increase awareness of the potential side effects that may require dose reductions. All patients enrolled in the Active Home Care project considered the information received on the oral regimen to be complete and exhaustive, and reported having been informed on the treatment-related side effects potentially associated with the medication they were taking. The percentage of patients reporting cancerand/or treatment-related symptoms decreased from $68.9 \%$ at baseline to $62.1 \%$ after 6 months. No serious adverse events were reported, and only nine hospital admissions were recorded, none of which was related to the underlying disease or treatment-associated adverse events. Overall, these results suggest that the management of symptoms and oral chemotherapy regimens by physicians and nurses was effective, and that communication between patients and health-care providers was adequate.

Compliance with treatment was high: all patients reported having taken their medications according to the indications provided by the physician. Adherence to long-term treatments is largely dependent on the perception of the individual risks, benefits, and costs of the intervention..$^{38}$ Almost $97 \%$ of patients considered home monitoring of compliance to treatment, patient health status, and possible adverse events by a nurse and/or an oncologist to be useful, and oral chemotherapy to be as effective as other treatments. Such patient perceptions, along with the improved ease of access to cancer care, may have contributed to the optimal adherence observed in this study. ${ }^{26}$ This finding is of particular relevance for the management of elderly patients with cancer, who often have comorbidities and other disabilities that require polypharmacy. In this setting, adherence to physicians' recommendations is highly important to avoid potentially dangerous drug-drug interactions. In addition, adherence among the elderly may be lower due to decreased social support and the increased incidence of cognitive issues. ${ }^{21}$ More than half (56\%) of patients enrolled in the Active Home Care project were elderly, which suggests that the initiative was equally effective in improving adherence to treatment in this patient population, as compared with younger patients. 
Overall, the high satisfaction with the program may be attributed to the increased convenience of home-delivered chemotherapy, which reduces the need for multiple trips to the hospital while nauseated or fatigued, and avoids inhospital waiting, enabling patients to benefit from the comfort of a familiar environment and less disruption to daily routine. The Active Home Care initiative also decreased the number of visits to primary-care physicians, which may result in reduced health-care costs. These findings are in accordance with previous studies showing that patients receiving home care make fewer visits to their general practitioner, have reduced contact with other health services, and have fewer inpatient days. ${ }^{39}$ In addition, home-based care models are associated with lower costs than inpatient hospital care. ${ }^{28}$

Home-based care may also have other advantages in terms of health-care and social costs. More than $80 \%$ of patients enrolled in the Active Home Care program would have needed to be accompanied to hospital visits by a family member or friend. This is particularly true for elderly patients, since access to treatment becomes more difficult with advancing age due to increased disability. Reducing the number of hospital visits would reduce both direct and indirect costs for caregivers, who often cover the costs of transportation and, in most cases, have to take time off from work. In addition, among caregivers, overall satisfaction with the program was even higher than among patients. Quality of life of caregivers is significantly affected by a loved one's cancer diagnosis. ${ }^{5}$ The home-based care model provides an optimal environment for the patient and his/her carers by reducing burdensome and time-consuming trips to hospital and by allowing patients and carers to maintain a normal lifestyle during treatment, which results in a positive impact on their quality of life.

The experience we report here confirms the results of a previous pilot study conducted by our group, ${ }^{40}$ which demonstrated that active home care may reduce patient accessing of cancer facilities by more than $98 \%$, promote better patient quality of life, and facilitate access to cancer care for those patients who require to be accompanied by carers. However, caution is needed when interpreting these findings, given the observational nature of this study and the lack of a control group. Further, a variety of cancer types and treatments were included, which could be regarded as a confounding factor, since different diseases and therapies may be associated with different outcomes, side effects, and response rates. Nevertheless, patients enrolled in the Active Home Care project were highly representative of the patient population attending the oncology referral centers involved in the initiative. In many cases, these patients lived some distance from their nearest oncology service, so our initiative allowed many individuals to overcome geographical barriers that would have prevented them from accessing adequate care. This experience is in accordance with the data reported in the literature on the integration of palliative care in the early stages of cancer and combines well with the appearance of the centrality of the oncologist in the management of the care of the patient. ${ }^{41}$ The self-reporting of adherence, which is subject to self-presentation bias, could be another limitation of this study. Although the reliability of the questionnaires used in this study will need to be confirmed in larger studies, self-report has high specificity and can provide information regarding the reasons for patient deviation from prescribed regimens. ${ }^{42}$

\section{Conclusion}

Home-based cancer treatment represents a new model of care that may lead to improvements in the quality of life of patients and caregivers, better adherence to treatment, and the effective management of therapy and cancer-related symptoms. Home-based cancer treatment may also optimize health-care-resource utilization, with a favorable impact on health-care expenditure. Further studies are needed to confirm the feasibility and benefits of this model.

\section{Acknowledgments}

Editorial assistance for the preparation of this manuscript was provided by Caterina Conte, MD, and Luca Giacomelli, $\mathrm{PhD}$, on behalf of Content Ed Net; this assistance was funded by Novartis.

\section{Disclosure}

The authors declare no conflicts of interest in this work.

\section{References}

1. Jemal A, Ward E, Thun M. Declining death rates reflect progress against cancer. PloS One. 2010;5(3):e9584.

2. Jemal A, Bray F, Center MM, Ferlay J, Ward E, Forman D. Global cancer statistics. CA Cancer J Clin. 2011;61(2):69-90.

3. Hwang SY, Chang SJ, Park BW. Does chemotherapy really affect the quality of life of women with breast cancer? J Breast Cancer. 2013; 16(2):229-235.

4. Reeve BB, Potosky AL, Smith AW, et al. Impact of cancer on healthrelated quality of life of older Americans. Journal of the National Cancer Institute. 2009;101(12):860-868.

5. Grant M, Sun V, Fujinami R, et al. Family caregiver burden, skills preparedness, and quality of life in non-small cell lung cancer. Oncol Nurs Forum. 2013;40(4):337-346.

6. Birner A. Pharmacology of oral chemotherapy agents. Clin J Oncol Nurs. 2003;7(6 Suppl):11-19.

7. O'Neill VJ, Twelves CJ. Oral cancer treatment: developments in chemotherapy and beyond. Br J Cancer. 2002;87(9):933-937. 
8. De Portu S, Mantovani LG, Ravaioli A, et al. Cost analysis of capecitabine vs 5-fluorouracil-based treatment for metastatic colorectal cancer patients. J Chemother. 2010;22(2):125-128.

9. Sanon M, Taylor DC, Parthan A, Coombs J, Paolantonio M, Sasane M. Cost-effectiveness of 3-years of adjuvant imatinib in gastrointestinal stromal tumors (GIST) in the United States. J Med Econ. 2013;16(1): $150-159$.

10. Cammà $\mathrm{C}$, Cabibbo $\mathrm{G}$, Petta $\mathrm{S}$, et al; WEF study group; SOFIA study group. Cost-effectiveness of sorafenib treatment in field practice for patients with hepatocellular carcinoma. Hepatology. 2013;57(3): 1046-1054.

11. Demetri GD, von Mehren M, Blanke CD, et al. Efficacy and safety of imatinib mesylate in advanced gastrointestinal stromal tumors. $N$ Engl J Med. 2002;347(7):472-480.

12. Rammohan A, Sathyanesan J, Rajendran K, et al. A gist of gastrointestinal stromal tumors: A review. World J Gastrointest Oncol. 2013; 5(6):102-112.

13. Weingart SN, Brown E, Bach PB, et al. NCCN Task Force Report: Oral chemotherapy. J Natl Compr Canc Netw. 2008;6 Suppl 3:S1-S14.

14. Hoff PM, Ansari R, Batist G, et al. Comparison of oral capecitabine versus intravenous fluorouracil plus leucovorin as first-line treatment in 605 patients with metastatic colorectal cancer: results of a randomized phase III study. J Clin Oncol. 2001;19(8):2282-2292.

15. O'Shaughnessy J, Miles D, Vukelja S, et al. Superior survival with capecitabine plus docetaxel combination therapy in anthracyclinepretreated patients with advanced breast cancer: phase III trial results. J Clin Oncol. 2002;20(12):2812-2823.

16. Van Cutsem E, Twelves C, Cassidy J, et al; Xeloda Colorectal Cancer Study Group. Oral capecitabine compared with intravenous fluorouracil plus leucovorin in patients with metastatic colorectal cancer: results of a large phase III study. J Clin Oncol. 2001;19(21):4097-4106.

17. Okines AF, Norman AR, McCloud P, Kang YK, Cunningham D. Metaanalysis of the REAL-2 and ML17032 trials: evaluating capecitabinebased combination chemotherapy and infused 5-fluorouracil-based combination chemotherapy for the treatment of advanced oesophagogastric cancer. Ann Oncol. 2009;20(9):1529-1534.

18. Effects of vinorelbine on quality of life and survival of elderly patients with advanced non-small-cell lung cancer. The Elderly Lung Cancer Vinorelbine Italian Study Group. J Natl Cancer Inst. 1999;91(1):66-72.

19. Le Chevalier T, Brisgand D, Douillard JY, et al. Randomized study of vinorelbine and cisplatin versus vindesine and cisplatin versus vinorelbine alone in advanced non-small-cell lung cancer: results of a European multicenter trial including 612 patients. J Clin Oncol. 1994; 12(2):360-367.

20. Freytes CO. Indications and complications of intravenous devices for chemotherapy. Curr Opin Oncol. 2000;12(4):303-307.

21. Partridge AH, Avorn J, Wang PS, Winer EP. Adherence to therapy with oral antineoplastic agents. J Natl Cancer Inst. 2002;94(9):652-661.

22. Ruddy K, Mayer E, Partridge A. Patient adherence and persistence with oral anticancer treatment. CA Cancer J Clin. 2009;59(1):56-66.

23. van Leeuwen RW, Brundel DH, Neef C, et al. Prevalence of potential drug-drug interactions in cancer patients treated with oral anticancer drugs. Br J Cancer. 2013;108(5):1071-1078.

24. Gebbia V, Bellavia G, Ferraù F, Valerio MR. Adherence, compliance and persistence to oral antineoplastic therapy: a review focused on chemotherapeutic and biologic agents. Expert Opin Drug Saf. 2012;11 Suppl 1:S49-S59.

Patient Preference and Adherence

\section{Publish your work in this journal}

Patient Preference and Adherence is an international, peer-reviewed, open access journal that focusing on the growing importance of patient preference and adherence throughout the therapeutic continuum. Patient satisfaction, acceptability, quality of life, compliance, persistence and their role in developing new therapeutic modalities and compounds to optimize
25. Lebovits AH, Strain JJ, Schleifer SJ, Tanaka JS, Bhardwaj S, Messe MR Patient noncompliance with self-administered chemotherapy. Cancer. 1990;65(1):17-22.

26. Osterberg L, Blaschke T. Adherence to medication. $N$ Engl J Med. 2005;353(5):487-497.

27. Tappenden P, Campbell F, Rawdin A, Wong R, Kalita N. The clinical effectiveness and cost-effectiveness of home-based, nurse-led health promotion for older people: a systematic review. Health Technol Assess. 2012;16(20):1-72.

28. Raphaël R, Yves D, Giselle C, Magali M, Odile CM. Cancer treatment at home or in the hospital: what are the costs for French public health insurance? Findings of a comprehensive-cancer centre. Health Policy. 2005;72(2):141-148.

29. Aaronson NK, Ahmedzai S, Bergman B, et al. The European Organization for Research and Treatment of Cancer QLQ-C30: a quality-of-life instrument for use in international clinical trials in oncology. $J$ Natl Cancer Inst. 1993;85(5):365-376.

30. Cella D, Davis K, Breitbart W, Curt G; Fatigue Coalition. Cancer-related fatigue: prevalence of proposed diagnostic criteria in a United States sample of cancer survivors. J Clin Oncol. 2001;19(14):3385-3391.

31. Rüffer JU, Flechtner H, Tralls P, et al; German Hodgkin Lymphoma Study Group. Fatigue in long-term survivors of Hodgkin's lymphoma; a report from the German Hodgkin Lymphoma Study Group (GHSG). Eur J Cancer. 2003;39(15):2179-2186.

32. van den Beuken-van Everdingen MH1, de Rijke JM, Kessels AG, Schouten HC, van Kleef M, Patijn J. Prevalence of pain in patients with cancer: a systematic review of the past 40 years. Ann Oncol. 2007;18(9): 1437-1449.

33. Laviano A, Meguid MM, Rossi-Fanelli F. Cancer anorexia: clinical implications, pathogenesis, and therapeutic strategies. Lancet Oncol. 2003;4(11):686-694.

34. Macciò A, Madeddu C, Mantovani G. Current pharmacotherapy options for cancer anorexia and cachexia. Expert Opin Pharmacother. 2012;13(17):2453-2472.

35. Peters L, Sellick K. Quality of life of cancer patients receiving inpatient and home-based palliative care. J Adv Nurs. 2006;53(5):524-533.

36. Borner MM, Dietrich D, Stupp R, et al. Phase II study of capecitabine and oxaliplatin in first- and second-line treatment of advanced or metastatic colorectal cancer. J Clin Oncol. 2002;20(7):1759-1766.

37. Liu G, Franssen E, Fitch MI, Warner E. Patient preferences for oral versus intravenous palliative chemotherapy. J Clin Oncol. 1997;15(1): $110-115$.

38. Love RR, Cameron L, Connell BL, Leventhal H. Symptoms associated with tamoxifen treatment in postmenopausal women. Arch Intern Med. 1991;151(9):1842-1847.

39. Molassiotis A, Brearley S, Saunders M, et al. Effectiveness of a home care nursing program in the symptom management of patients with colorectal and breast cancer receiving oral chemotherapy: a randomized, controlled trial. J Clin Oncol. 2009;27(36):6191-6198.

40. Bordonaro S, Raiti F, Di Mari A, et al. Active home-based cancer treatment. J Multidiscip Healthc. 2012;5:137-143.

41. Temel JS, Greer JA, Muzikansky A, et al. Early palliative care for patients with metastatic non-small-cell lung cancer. NEngl J Med. 2010; 363(8):733-742.

42. Bhattacharya D, Easthall C, Willoughby KA, Small M, Watson S. Capecitabine non-adherence: exploration of magnitude, nature and contributing factors. J Oncol Pharm Pract. 2012;18(3):333-342. 